\title{
Equipartitioning Common Domains of Non-Atomic Measures
}

\author{
Theodore P. Hill
}

Department of Mathematics, University of Hawaii, Honolulu, HI 96822, USA

\section{$\S 1$. Introduction}

Let $h\left(a_{1}, \ldots, a_{n}\right)$ denote the harmonic mean $n\left(a_{1}^{-1}+\ldots+a_{n}^{-1}\right)^{-1}$ of positive numbers $a_{1}, \ldots, a_{n}$, and extend this definition (by continuity) to the non-negative case by setting $h\left(a_{1}, \ldots, a_{n}\right)=0$ if $a_{i}=0$ for some $i \leqq n$. Recall that a measure $\mu$ is non-atomic if every measurable set $A$ of strictly positive measure has a measurable subset $B \subseteq A$ satisfying $\mu(A)>\mu(B)>0$. A measurable partition of a measure space $(X, \mathscr{F})$ is a collection of disjoint subsets $A_{1}, \ldots, A_{n}$ of $X$ satisfying $A_{i} \in \mathscr{F}$

for all $i$, and $\bigcup_{i=1}^{n} A_{i}=X$. Throughout this paper, "measure" means "countably additive, non-negative measure", and $\|\mu\|$ is the total mass (or total variation) of the measure $\mu$; the main purpose is to prove the following theorem.

Theorem 1.1. Let $\mu_{1}, \mu_{2}, \ldots, \mu_{n}$ be non-atomic finite measures on a measure space $(X, \mathscr{F})$. Then there is a measurable partition $A_{1}, \ldots, A_{n}$ of $X$ satisfying

$$
\mu_{i}\left(A_{i}\right) \geqq n^{-1} h\left(\left\|\mu_{1}\right\|, \ldots,\left\|\mu_{n}\right\|\right) \quad \text { for all } i,
$$

and this bound is best possible. Moreover, if $\left\|\mu_{i}\right\|>0$ for all $i$, then:

(2a) if $\mu_{i} /\left\|\mu_{i}\right\|=\mu_{j} /\left\|\mu_{j}\right\|$ for all $i, j \leqq n$, then every partition satisfying (1) holds with equality for all $i$; and

(2b) otherwise, the $\left\{A_{i}\right\}$ may be chosen so that strict inequality holds in (1) for all $i$.

Theorem 1.1 has an interesting "distribution of value" or "fair-division" interpretation. Suppose an object, such as a cake or piece of land, is to be divided among $n$ people whose values of the object may differ. What is the largest $c$ possible so that each person may receive a piece he values at least $c$, according to his own values? Theorem 1.1 essentially says that if the values vary continuously (e.g., pieces of zero volume are worth nothing), then $c$ is at least $1 / n$ times the harmonic mean of the total values assigned by each person, and that in general it is not possible to do better. 
Corollary 1.2 (Dubins and Spanier [2]). Let $\mu_{1}, \ldots, \mu_{n}$ be non-atomic probability measures on a measure space $(X, \mathscr{F})$. Then there is a measurable partition $A_{1}, \ldots, A_{n}$ of $X$ satisfying

$$
\mu_{i}\left(A_{i}\right) \geqq 1 / n \quad \text { for all } i,
$$

and if $\mu_{i} \neq \mu_{j}$ for some $i \neq j$, then strict inequality in (3) for all $i$ may be attained.

(Actually, Dubins and Spanier showed more: for example, $\mu_{i}\left(A_{i}\right) \geqq p_{i}$ may be attained for any $p_{i} \geqq 0, \sum_{i=1}^{n} p_{i}=1$.)

The question underlying (3) dates back to Steinhaus [7], and the reader is referred to [2] for a number of interesting related results many of which are based on Lyapounov's convexity theorem. A proof of Theorem 1.1 can also be given using the convexity theorem, but the argument below is based on a recent $L_{1}(0,1)$ inequality of Dor.

\section{§2. Proof of Main Theorem}

For measures $m_{1}, m_{2}, \ldots, m_{n}$ on $(X, \mathscr{F})$, let $\bigvee_{i=1}^{n} m_{i}$ denote the smallest measure which dominates each of the $\left\{m_{i}\right\}$, that is, for each $A \in \mathscr{F}$,

$$
\left(\bigvee_{i=1}^{n} m_{i}\right)(A)=\sup \left\{\sum_{i=1}^{n} m_{i}\left(B_{i}\right): B_{1}, \ldots, B_{n} \text { is a measurable partition of } A\right\} \text {. }
$$

The next proposition provides the key step in the proof; it is a standard generalization, via the non-atomic separable-measure-algebra isomorphism theorem (e.g. [4] Theorem C, p. 173), of a result of Dor ([1], p. 472) who stated it only for $L_{1}(0,1)$.

Proposition 2.1. Let $\mu_{1}, \mu_{2}, \ldots, \mu_{n}$ be non-atomic finite measures on a measure space $(X, \mathscr{F})$, and let $c \geqq 0$. If

$$
\left\|\bigvee_{i=1}^{n} \alpha_{i} \mu_{i}\right\| \geqq c \sum_{i=1}^{n} \alpha_{i} \quad \text { for all } \alpha_{1}, \ldots, \alpha_{n} \geqq 0,
$$

then there are disjoint measurable sets $A_{1}, \ldots, A_{n} \in \mathscr{F}$ satisfying

$$
\mu_{i}\left(A_{i}\right) \geqq c \quad \text { for all } i \leqq n .
$$

The proof of Theorem 1.1 will also use three short lemmas; the first lemma must be known, but as no reference is known to the author, a proof is given.

For each positive integer $n$, let $\prod_{n}$ denote the space of probabilities on $n$ points, that is $\prod_{n}=\left\{\vec{p}=\left(p_{1}, \ldots, p_{n}\right) \in \mathbb{R}^{n}: p_{i} \geqq 0\right.$ for all $i$, and $\left.\sum_{i=1}^{n} p_{i}=1\right\}$. 
Lemma 2.2. If $a_{1}, \ldots, a_{n}$ are strictly positive, then

$$
\inf _{\vec{p} \in \Pi_{n}} \max _{i \leqq n}\left\{p_{i} a_{i}\right\}=n^{-1} h\left(a_{1}, \ldots, a_{n}\right)=\sup _{\vec{p} \in \Pi_{n}} \min _{i \leqq n}\left\{p_{i} a_{i}\right\},
$$

and both equalities are attained only for $\vec{p}=\left(p_{1}^{*}, \ldots, p_{n}^{*}\right)$ where $p_{i}^{*}$ $=a_{i}^{-1} n^{-1} h\left(a_{1}, \ldots, a_{n}\right)$.

Proof. By the compactness of $\prod_{n}$ and the continuity in $\vec{p}$ of both $\min _{i \leqq n}\left\{p_{i} a_{i}\right\}$ and $\max _{i \leqq n}\left\{p_{i} a_{i}\right\}$, the inf and sup in (6) are both attained. An easy redistribution of mass argument then shows the inf and sup are each attained only when $p_{1} a_{1}$ $=\ldots=p_{n} a_{n} ;$ substitution into the equation $\sum_{i=1}^{n} p_{i}=1$ yields the desired conclusion.

Lemma 2.3. Let $\mu_{1}, \ldots, \mu_{n}$ be finite measures. Then

$$
\begin{gathered}
\left\|\bigvee_{i=1}^{n} p_{i} \mu_{i}\right\| \geqq \max _{i \leqq n}\left\|p_{i} \mu_{i}\right\| \geqq n^{-1} h\left(\left\|\mu_{1}\right\|, \ldots,\left\|\mu_{n}\right\|\right) \\
\text { for all }\left(p_{1}, \ldots, p_{n}\right) \in \prod_{n} .
\end{gathered}
$$

Proof. The first inequality in (7) is elementary, and the second follows by Lemma 2.2 with $a_{i}=\left\|\mu_{i}\right\|$.

Lemma 2.4. Suppose $\left\|\mu_{i}\right\|>0$ for all $i \leqq n$. If $\left\|\bigvee_{i=1}^{n} p_{i} \mu_{i}\right\|=n^{-1} h\left(\left\|\mu_{1}\right\|, \ldots,\left\|\mu_{n}\right\|\right)$ for some $\left(p_{1}, \ldots, p_{n}\right) \in \prod_{n}$, then $p_{i}=\left\|\mu_{i}\right\|^{-1} n^{-1} h\left(\left\|\mu_{1}\right\|, \ldots,\left\|\mu_{n}\right\|\right)$ for all $i \leqq n$, and $p_{i} \mu_{i}=p_{j} \mu_{j}$ for all $i, j \leqq n$.

Proof. By (7), $\max \left\|p_{i} \mu_{i}\right\|=n^{-1} h\left(\left\|\mu_{1}\right\|, \ldots,\left\|\mu_{n}\right\|\right)$, and by Lemma 2.2 this implies $p_{i}=\left\|\mu_{i}\right\|^{-1} n^{-1} h\left(\left\|\mu_{1}\right\|, \ldots,\left\|\mu_{n}\right\|\right)$ for all $i \leqq n$. Hence

$$
\left\|\bigvee_{i=1}^{n} p_{i} \mu_{i}\right\|=\left\|p_{1} \mu_{1}\right\|=\ldots=\left\|p_{n} \mu_{n}\right\|,
$$

which in turn implies $p_{i} \mu_{i}=p_{j} \mu_{j}$ for all $i, j \leqq n$.

Proof of Theorem 1.1. If $\left\|\mu_{i}\right\|=0$ for some $i$, then $h\left(\left\|\mu_{1}\right\|, \ldots,\left\|\mu_{n}\right\|\right)=0$, and (1) follows immediately; that this bound is best possible in this case is clear.

For the remainder of the proof, suppose $\left\|\mu_{i}\right\|>0$ for all $i=1, \ldots, n$. Lemma 2.3 implies that (4) is satisfied with $c=n^{-1} h\left(\left\|\mu_{1}\right\|, \ldots,\left\|\mu_{n}\right\|\right)$, (the case $\alpha_{1}$ $=\ldots=\alpha_{n}=0$ being trivial), and (1) follows from Proposition 2.1; that this bound is best possible will follow from (2a).

Suppose $\mu_{i} /\left\|\mu_{i}\right\|=\mu_{j} /\left\|\mu_{j}\right\|$ for all $i, j \leqq n$, and let $A_{1}, \ldots, A_{n}$ be a measurable partition of $X$ satisfying (1). Then for all $i \leqq n$, 


$$
\begin{aligned}
\mu_{i}\left(A_{i}\right) & =\sum_{j=1}^{n} \mu_{i}\left(A_{j}\right)-\sum_{j \neq i}^{n} \mu_{i}\left(A_{j}\right) \\
& =\left\|\mu_{i}\right\|-\sum_{j \neq i}^{n}\left\|\mu_{i}\right\|\left\|\mu_{j}\right\|^{-1} \mu_{j}\left(A_{j}\right) \\
& \leqq\left\|\mu_{i}\right\|\left(1-n^{-1} h\left(\left\|\mu_{1}\right\|, \ldots,\left\|\mu_{n}\right\|\right) \sum_{j \neq i}^{n}\left\|\mu_{j}\right\|^{-1}\right) \\
& =n^{-1} h\left(\left\|\mu_{1}\right\|, \ldots,\left\|\mu_{n}\right\|\right),
\end{aligned}
$$

so by (1) equality holds throughout, which establishes (2a).

Finally, suppose $\mu_{i} /\left\|\mu_{i}\right\| \neq \mu_{j} /\left\|\mu_{j}\right\|$ for some $i \neq j$. By Lemmas 2.3 and 2.4, $\left\|\bigvee_{i=1}^{n} p_{i} \mu_{i}\right\|>n^{-1} h\left(\left\|\mu_{1}\right\|, \ldots,\left\|\mu_{n}\right\|\right)$ for all $\left(p_{1}, \ldots, p_{n}\right) \in \prod_{n}$. The compactness of $\prod_{n}$ and continuity of $\left\|\bigvee_{i=1}^{n} p_{i} \mu_{i}\right\|$ in $\vec{p}$ then imply the existence of a $c>n^{-1} h\left(\left\|\mu_{1}\right\|, \ldots\right.$, $\left.\left\|\mu_{n}\right\|\right)$ satisfying $\left\|\bigvee_{i=1}^{n} p_{i} \mu_{i}\right\|>c$ for all $\left(p_{1}, \ldots, p_{n}\right) \in \prod_{n}$, which by Proposition 2.1 completes the proof of $(2 \mathrm{~b})$.

\section{§3. Structured Domains}

When $\mu_{1}, \ldots, \mu_{n}$ are all probability measures, applications of inequalities of the type in Corollary 1.2 have been made to statistical hypotheses testing (see for example $[3,6]$ ). If the domain in question has a particular structure (e.g., as in [5]), then one may ask whether there is a measurable partition $\left\{A_{i}\right\}$ with the same structure satisfying (1) or (3). The following theorem is a typical result along these lines; its proof uses an idea in [5] and is given here primarily as an application of the strict inequality conclusion (2b) of Theorem 1.1.

Theorem 3.1. Suppose $f_{1}, \ldots, f_{n}$ are continuous non-negative functions defined on a polygon $P \subset \mathbb{R}^{2}$. Then there is a polygonal decomposition $P_{1}, \ldots, P_{n}$ of $P$ satisfying

$$
\int_{P_{i}} f_{i} \geqq\left[\left(\int_{P} f_{1}\right)^{-1}+\ldots+\left(\int_{P} f_{n}\right)^{-1}\right]^{-1} \quad \text { for all } i \leqq n,
$$

and this bound is best possible.

Sketch of Proof. If $f_{i} \equiv 0$ for some $i$, any polygonal decomposition of $P$ will suffice, so assume $\int_{P} f_{i}>0$ for all $i \leqq n$. Suppose first that $f_{i} / \int_{P} f_{i}=f_{j} / \int_{P} f_{j}$ for all $i, j \leqq n$. Take as $P_{1}$ any polygon contained in $P$ for which $P \backslash P_{1}$ is also a polygon and $\int_{P_{1}} f_{1}=\left[\left(\int_{P} f_{1}\right)^{-1}+\ldots+\left(\int_{P} f_{n}\right)^{-1}\right]^{-1}$. Continue with $P_{2}$ in $P \backslash P_{1}$, and so on; since the functions are proportional, (8) is seen to be satisfied with equality throughout. On the other hand, if $f_{i} / \int_{P} f_{i} \neq f_{j} / \int_{P} f_{j}$ for some $i \neq j$, then use 
Theorem 1.1(2b) to infer the existence of a Borel partition $A_{1}, \ldots, A_{n}$ of $P$ satisfying

$$
\int_{A_{i}} f_{i}>\left[\left(\int_{P} f_{1}\right)^{-1}+\ldots+\left(\int_{P} f_{n}\right)^{-1}\right]^{-1} \quad \text { for all } i \leqq n .
$$

Using this strict inequality, approximate the $\left\{A_{i}\right\}$ simultaneously by disjoint polygons $\left\{P_{i}\right\}$ in $P$ in such a way as to retain the inequality.

\section{§4. Remarks}

Clearly the conclusion of Theorem 1.1 may fail if the measures have atoms (the worst case being when each $\mu_{i}$ is the dirac delta measure on the same point $x \in X$ ), and as the next example shows, the conclusion (1) may also fail if the requirement of finiteness of the measures is dropped (where $h$ is defined using $0^{-1}=\infty$ and $\infty^{-1}=0$ ).

Example 4.1. Let $\mu_{1}$ be Lebesgue measure on $[0,1]$, and let $\mu_{2}$ be given by $\mu_{2}(A)=\int_{A} f d \mu_{1}$ where $f(x)=1 / x$ for all $x \in(0,1)$. Then $\left\|\mu_{1}\right\|=1,\left\|\mu_{2}\right\|=\infty$, and $\left(\left\|\mu_{1}\right\|^{-1}+\left\|\mu_{2}\right\|^{-1}\right)^{-1}=1$, but for any measurable set $A$, either $\mu_{1}(A)<1$ or $\mu_{2}\left(A^{c}\right)=0$, so (1) fails.

If the requirement of non-negativity of the measures is dropped, it is also easy to see that inequality (1) may fail, whether $\|\mu\|$ is interpreted either as the total variation or as the total mass of $\mu$.

Acknowledgements. The author is grateful to Stephen Demko for bringing the Dor result to his attention, to Carl Spruill for several conversations, and to the referee for several suggestions.

\section{Bibliography}

1. Dor, L.: On projections in $L_{1}$. Ann. Math. 102, 463-474 (1975)

2. Dubins, L., Spanier, E.: How to cut a cake fairly. Am. Math. Mon. 68, 1-17 (1961)

3. Feller, W.: Note on regions similar to the sample space. Statistical Research Memoirs, Univ. of London, 116-125 (1938)

4. Halmos, P.: Measure Theory. New York: Van Nostrand 1950

5. Hill, T.: Determining a fair border. Am. Math. Mon. 90, 438-442 (1983)

6. Neyman, J., Pearson, E.: On the problem of the most efficient tests of statistical hypotheses. Philos. Trans. R. Soc. Lond., Ser. A 231, 289-337 (1933)

7. Steinhaus, H.: Sur la division pragmatique. Econometrica (Supplement) 17, 315-319 (1949) 